\title{
SERUM GALECTINS ARE INCREASED IN PATIENTS WITH GRAVES' DISEASE HYPERTHYROIDISM
}

\author{
A Ramos-Leví ${ }^{1}$, A Serrano${ }^{1}$, M Sampedro-Núñez ${ }^{1}$, A Vicuña ${ }^{1}$, A Rodríguez-Muñoz ${ }^{1}$, R Martínez ${ }^{1}$, \\ H de la Fuente ${ }^{2}$, M Marazuela ${ }^{1}$ \\ ${ }^{1}$ Department of Endocrinology and Nutrition and ${ }^{2}$ Department of Clinical Immunology; Hospital Universitario de la \\ Princesa, Instituto de Investigación Sanitaria Princesa, Universidad Autónoma de Madrid, SPAIN.
}

\section{INTRODUCTION}

Patients with autoimmune thyroid disease (AITD) exhibit aberrant immune-regulatory mechanisms. Galectins (Gal) are a family of glycan-binding proteins, which have been involved in immune regulation. However, the association of Gal with AITD remains unknown. In this study, we evaluated serum Gal-1 and Gal-9 in patients with AITD.

\section{MATERIALS AND METHODS}

We studied peripheral blood samples from 31 patients with Graves' disease (GD) (14 with untreated hyperthyroidism, 9 euthyroid with treatment, 8 with iatrogenic hypothyroidism), 26 Hashimoto's thyroiditis (HT) (20 hypothyroid, 6 euthyroid with treatment), 12 non-GD hyperthyroid patients (NG) and 24 healthy controls were studied.

Serum levels of free thyroxine (FT4), TSH, thyroid antibodies (Ab) (anti-thyroglobulin, $\operatorname{TgAb}$, anti-thyroid peroxidase (TPOAb), and TSH receptor antibodies, TSHRAb), Gal-1 and Gal-9 were measured on the same day. FT4 was measured by radioimmuneanalysis (RIA); TSH, Tg-Ab, TPO-Ab and TSHR-Ab by immunoradiometric assays; Gal-1 and Gal-9 by ELISA.

Patients were grouped according to clinical diagnosis and thyroidal status.

\section{RESULTS}

Serum levels of Gal-1 (ng/mL) (figure 1A) and Gal-9 $(\mathrm{pg} / \mathrm{mL}$ ) (figure 1B) were significantly increased in GD (3.756 and 8.582, respectively), HT $(3.085,9.188)$ and NG $(2.822,8.983)$, in comparison to controls $(1.508,7.323), p<0.05$. No significant differences in Gal levels were found between the first three groups.

Patients with hyperthyroidism (both GD and NG) had higher Gal-9 levels than euthyroid AITD patients (figure 2), although there were no differences in Gal-1. In fact, in GD, we observed a direct correlation between Gal-9 and FT4 ( $r=0.517, p=0.006)$ ) (figure 3a), and an inverse correlation between Gal-9 and TSH ( $r=-0.478, p=0.007)$ (figure 3b).

We did not find an association between Gal-9 and Ab levels in either group of AITD, or with the presence of Graves' orbitopathy in patinets with GD. Conversely, an association between Gal-1 and TPO-Ab levels was found in HT ( $r=-0.512, p=0.038)$. Antithyroid treatment reduced Gal-1 levels in patients with GD (figure 4).
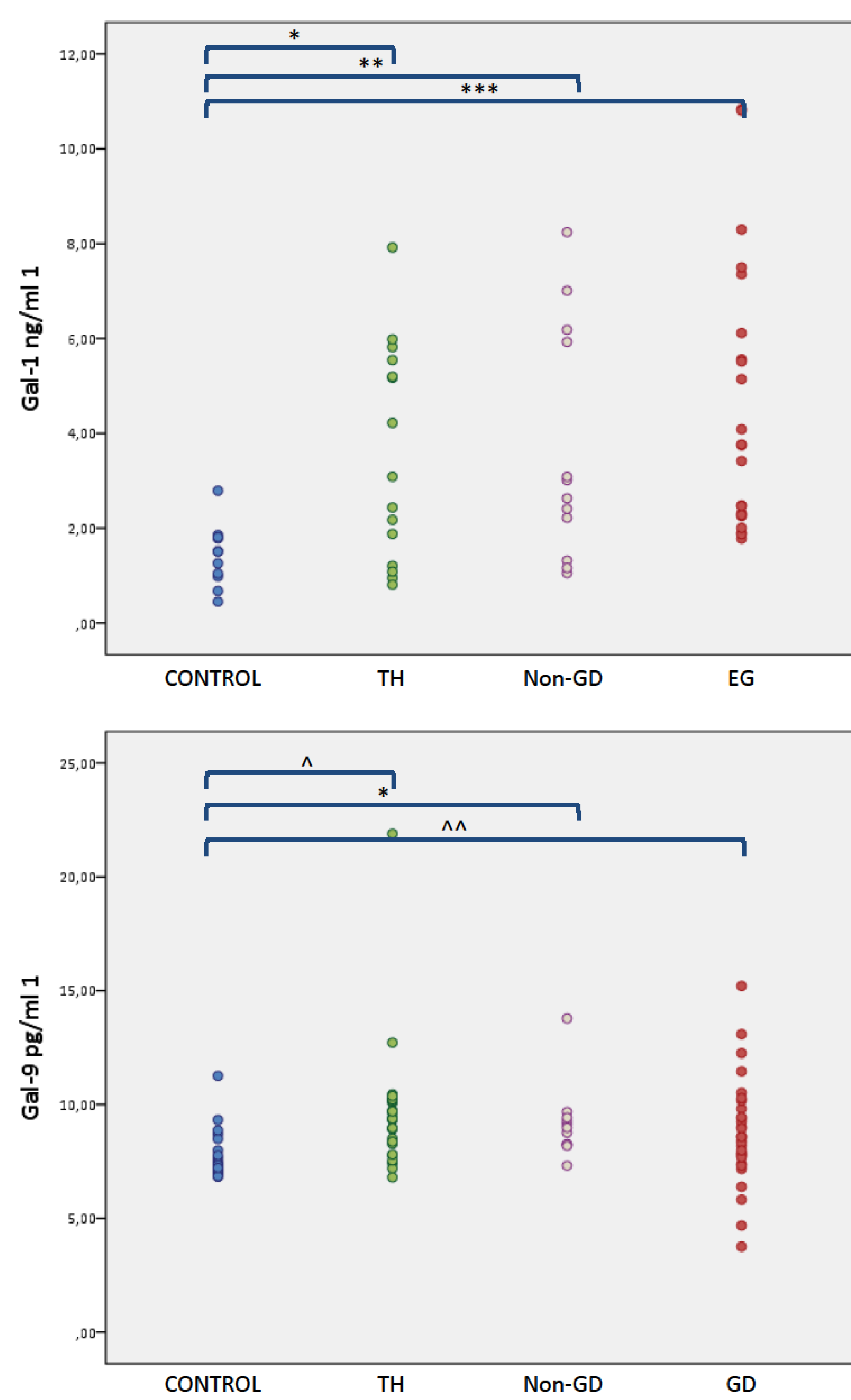

Figure 1. Serum levels of Gal 1 (A) and Gal 9 (B) from patients with $H T$, GD and non-GD, an controls. Paired comparisons (U-Man Whitney): ${ }^{*} \mathrm{p}=0.000 ;{ }^{* *} \mathrm{p}=0.013 ;{ }^{* * *} \mathrm{p}=0.007 ;{ }^{\wedge} \mathrm{p}=0.006 ;$ $\wedge \wedge \mathrm{p}=0.001$. Comparison between the 4 groups (Kruskal Wallis): $\mathrm{p}=0.001$ for Gal 1 and $\mathrm{p}=0.000$ for Gal 9

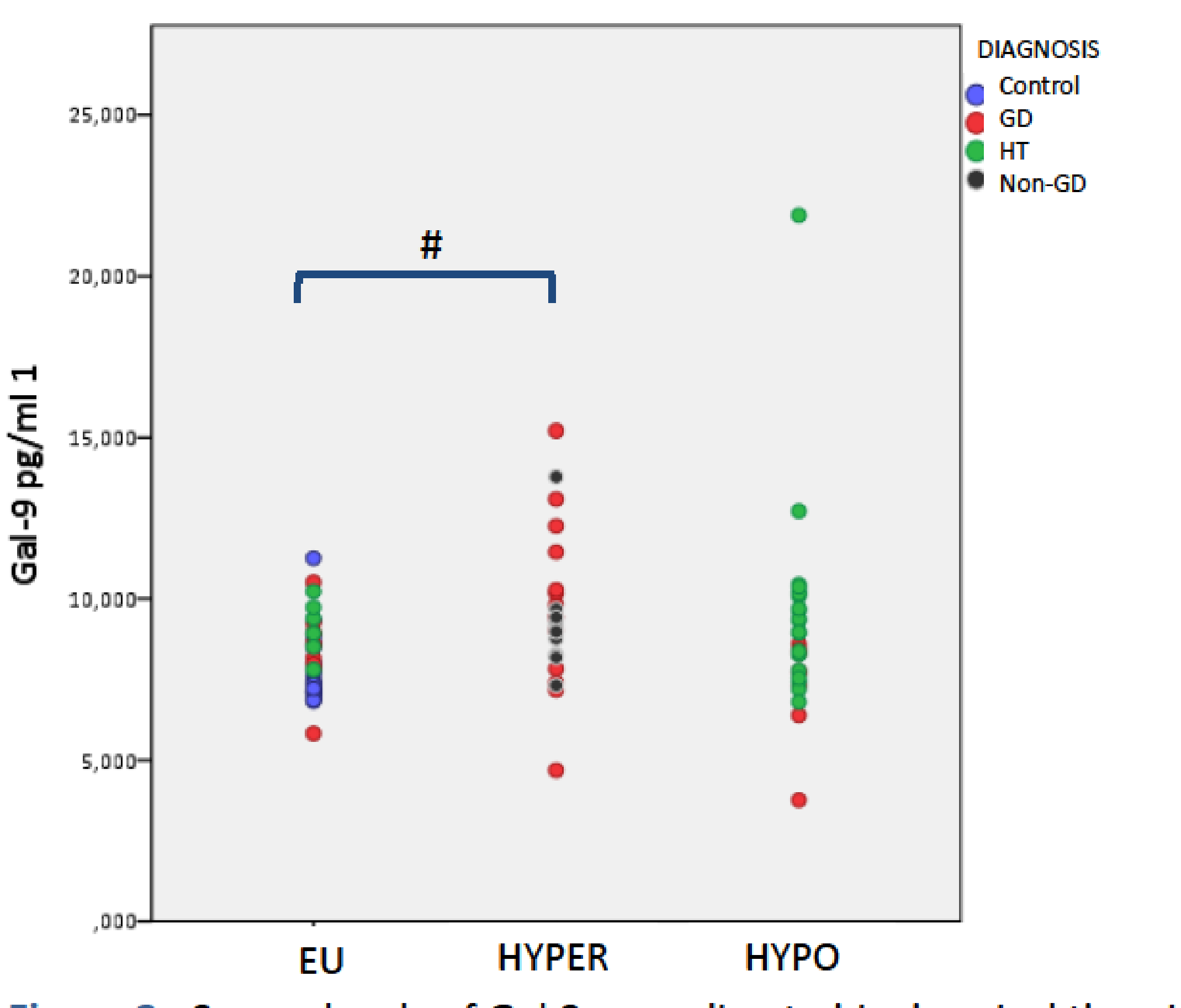

Figure 2. Serum levels of Gal 9 according to biochemical thyroid status. Kruskal Wallis for comparison between the 3 groups $\mathrm{p}=0.004$. Paired comparison EU vs HYPER $\# \mathrm{p}=0.001$

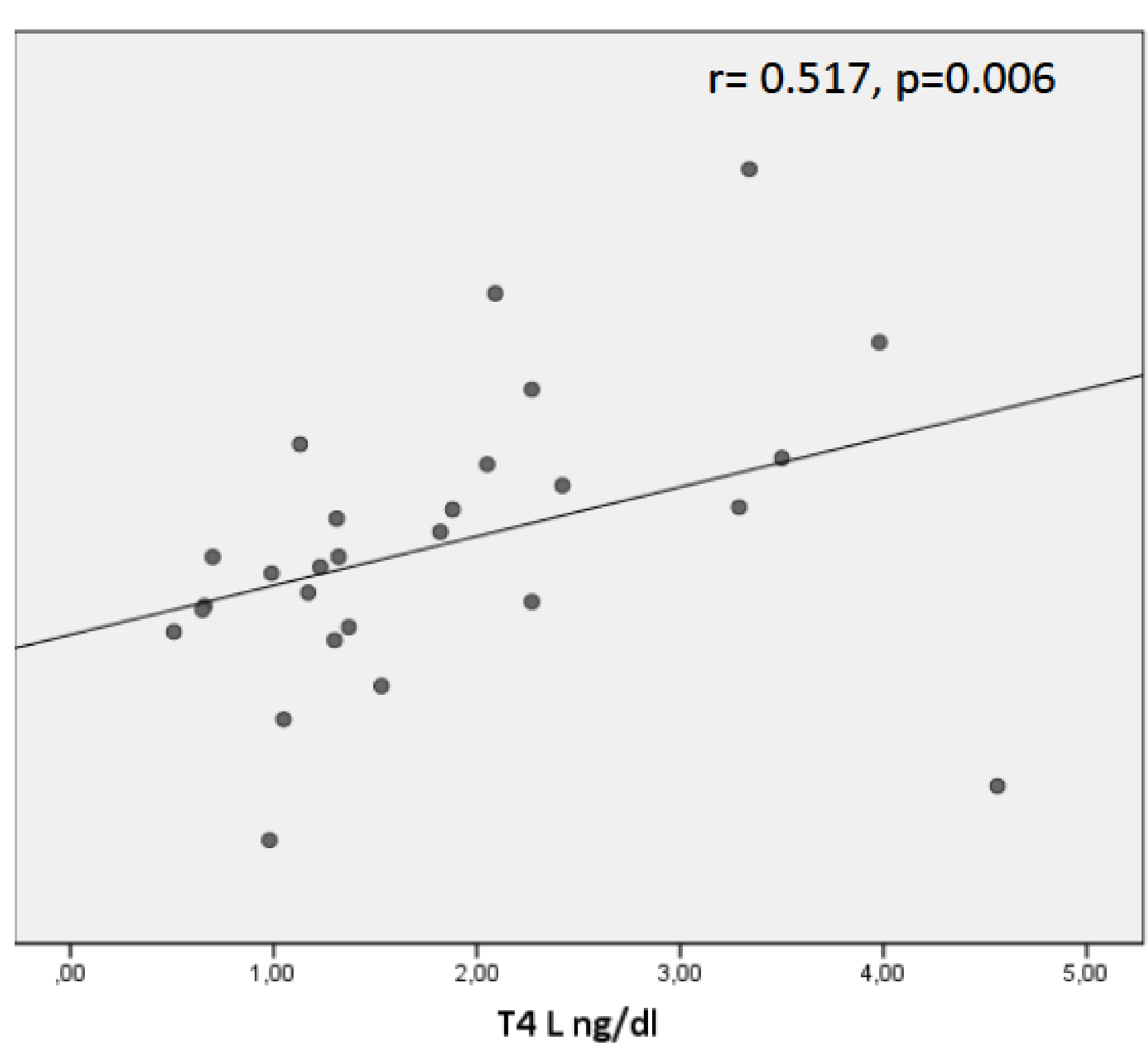

Figure 3. Bivariate correlations (Spearman) of Gal 9 with FT4 (A) and TSH (B) in patients with GD

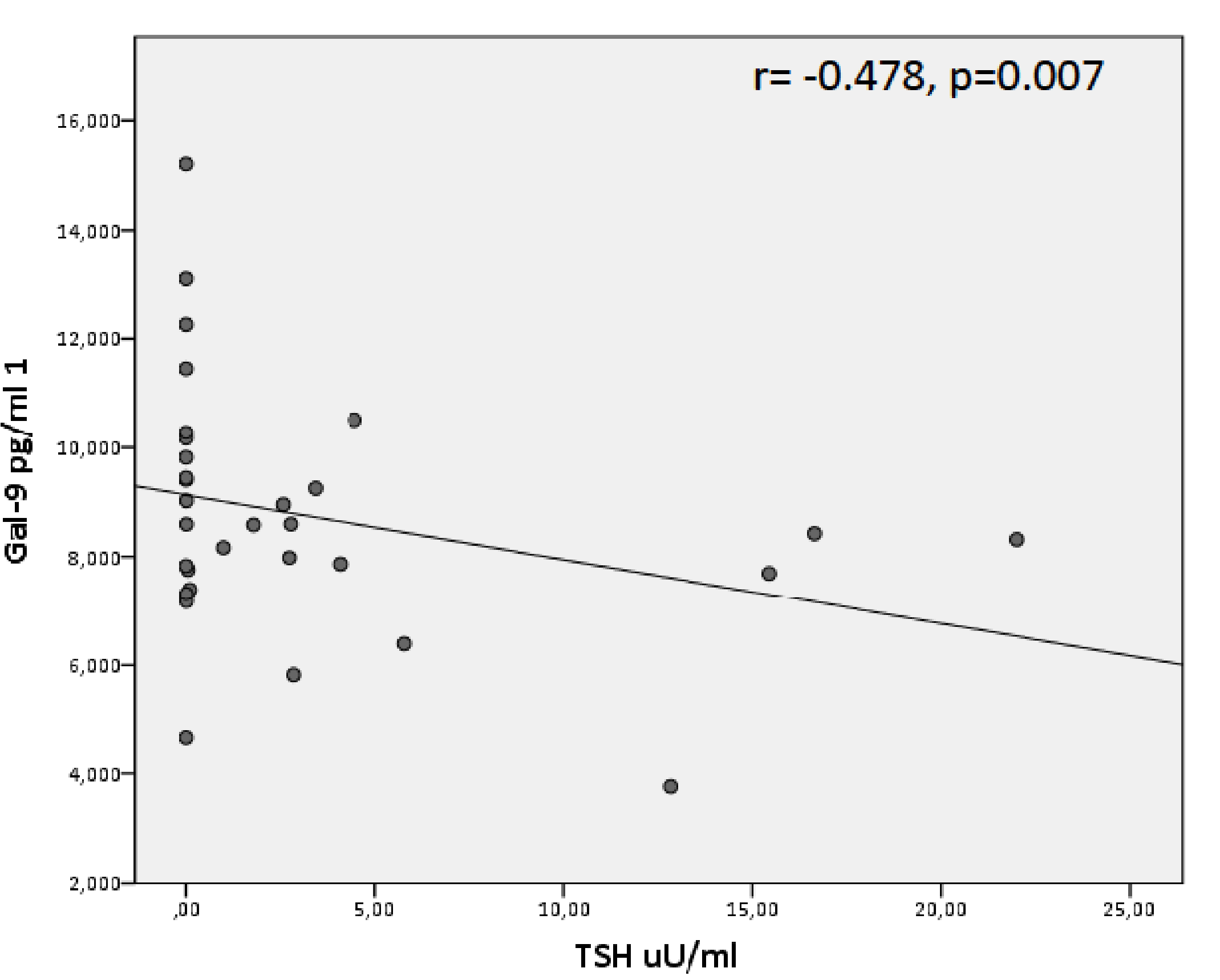

Figure 4. Serum levels of $G$ a 1 when in hyperthyroidism (left), and when euthyroid with treatment (right). Wilcoxon's test for related samples $\mathrm{p}=0.05$

\section{CONCLUSIONS}

Galectins may be involved in the severity and pathogenesis of AITD, and could potentially be used as a diagnostic and therapeutic marker.

ACKNOWLEDGEMENTS: Grants form Fundación SEEN Award for Young Endocrinologists, FIS 13/1414 and PIE 13/41. 